BLS 35, No 1 2009. DOI: http://dx.doi.org/10.3765/bls.v35i1.3600

(published by the Berkeley Linguistics Society and the Linguistic Society of America)

\title{
Temporal Interpretation and Tenselessness: The Case of Tagalog
}

\author{
JERUEN E. DERY \\ University at Buffalo (SUNY)
}

\section{Introduction}

One of the tasks required to understand narrative discourse is ordering events described by the sentences in the narrative. Previous research has claimed that at least three factors affect the way the order of events is determined. One of these factors is the "anaphoricity" of tense, which means that the tense of a sentence refers back to the time just after the event described by the previous sentence, or that it refers back to the time at which the state that is described by the previous sentence holds. This view was first proposed by Partee (1973) and further developed in Partee (1984). To illustrate, consider (1).

\section{(1) Sheila had a party last Friday and Sam got drunk.}

Partee argues that most uses of tenses are referential. In the second sentence in (1), the past tense does not refer back to any random time before the time of utterance. Rather, it refers back specifically to the vicinity of the time in which the state that is described by the previous clause holds. This analysis, which posits that tenses refer back to some other time that is given by previous event and state descriptions, is the main gist of models of discourse interpretation that make use of the notion of temporal anaphora. Discourse interpretation models such as Discourse Representation Theory have made use of the notion of temporal anaphora in explaining the temporal relationship of events and states in narrative discourse (Kamp \& Reyle 1993). Through temporal anaphoricity, the temporal ordering of events is established.

Another possible factor that affects the interpretation of event order is world knowledge. Speakers usually have knowledge of how the world operates and functions; i.e., they know that certain events tend to trigger other events, e.g., an event involving a barking dog may be followed by an event of a running cat. Speakers usually have knowledge of the cause-and-effect relations that hold between different events in real life. Speakers may use this world knowledge to determine the temporal order of events described by sequences of sentences. To illustrate, consider (2) and (3). 
(2) The baby cried. Bill pinched him.

(3) The baby cried. Bill consoled him.

Most speakers would assume that pinching a baby would most probably cause the baby to cry and infer a cause-and-effect relation between the two events. And speakers would also reason that causes happen before their intended effects, therefore, in (2), the event described by the second sentence occurs before the event described by the first sentence, resulting in an event ordering that is the reverse of the sentences' order. On the other hand, a crying event could also probably trigger a consoling event. Therefore, in (3), based on one's knowledge of the world, the event described by the first sentence would be interpreted as happening before the event described by the second sentence.

A third factor that influences the temporal interpretation of narrative discourses is the presentational order of sentences. For example, consider the discourses in (4) and (5).

(4) John and Mary got married. Mary moved into John's house.

(5) Mary moved into John's house. John and Mary got married.

In both examples, there is a tendency to interpret the sequence of events as identical to the order in which they are presented. In other words, the first sentence corresponds to the first event, and the second sentence corresponds to the second event. Thus, the order of presentation matters, since the temporal ordering of the two events differs as a function of the order of presentation. In this study, discourse which receives a temporal interpretation identical to the order of presentation will be labeled as "canonical order" discourse, while those which receive a temporal interpretation in which the order of presentation and event order do not match will be labeled as "non-canonical order" discourse. If a language does not have tense, it may be the case that non-canonical order is more frequent than in tensed languages, since the second sentence's temporal interpretation is not tied to the first sentence's temporal interpretation via a DRT-style interpretation of tense. Lack of tense may result in a less frequent iconic interpretation of events in discourse.

The lack of tense may also cause other factors such as aspect marking to have a larger role in the temporal interpretation of discourse. Aspect refers to the "different ways of viewing the internal temporal constituency of a situation," as defined in Comrie (1976). Aspect marking can signal whether an event is ongoing (progressive), completed (perfective), or about to occur (prospective). The fact that a sentence marks the described situation's aspect can also be a source of information when locating a certain event in time.

This study aims to see what the factors are that affect temporal interpretation if a language lacks tense, and therefore notions of temporal anaphoricity are not applicable. I investigate how other factors such as world knowledge, presentational order of sentences, and aspect marking contribute to the task of the tempo- 
ral interpretation of narrative discourse, and try to present a model of other elements that come into play if tense is unavailable as a resource for temporal interpretation. It is hypothesized that in a tenseless language, aspect markers can act as sources of temporal information without invoking anaphoricity per se. It is also hypothesized that the available factors in a tenseless language do not contribute equally to the task of temporal interpretation. Specifically, it is hypothesized that in a tenseless language, world knowledge may play a larger role in determining how events are ordered with respect to each other, due to the fact that lack of tense blocks a temporal interpretation as posited by Discourse Representation Theory (Kamp and Reyle 1993:497). To test these hypotheses regarding temporal interpretation in a tenseless language, I conducted an experiment on Tagalog, a tenseless language, which manipulated three factors: aspect marking, presentational order, and world knowledge.

\section{Experiment}

The experiment consisted of a norming study conducted with English speakers, and a main experiment conducted with Tagalog speakers.

\subsection{Norming Study}

Prior to conducting an experiment with Tagalog speakers, a norming study was conducted with English speakers, in order to determine the event-order bias of the stimulus sets. The norming study measured the likelihood of events happening before or after another event. It was conducted with English speakers, while the experiment was conducted with Tagalog speakers, assuming that the relevant portions of the world in an English-speaking area behave similarly to corresponding portions of the world in a Tagalog-speaking area.

For the norming study, participants evaluated the likelihood that one event will happen before or after another event in a two-event discourse. For example, in (6), one set of participants were asked to rate the likelihood that the event described in the sentence Bill pushed John preceded the event described in the sentence John fell, while another set of participants were asked to rate the likelihood that the event described in the sentence John fell preceded the event described in the sentence Bill pushed John. This was done in order to classify stimuli as to whether they had a preferred order of interpretation or not.

(6) Bill pushed John. John fell.

(7) John fell. Bill pushed John.

(8) John sliced the pizza. Mary poured the drinks.

(9) Mary poured the drinks. John sliced the pizza.

A total of 154 test items were constructed. Each of these test items consisted of two sentences, marked A and B. Each of the 154 test items had two versions as all test items were presented in canonical and non-canonical order. "Canonical order" here refers to stimuli in which the event that has a high probability of 
occurring first is described in the first sentence, as in (6), while "non-canonical order" refers to stimuli in which the event that has a high probability of occurring first is described in the second sentence, as in (7). Half of the stimuli were predicted to involve a preferred order of interpretation, such as (6) and (7), while the other half of the stimuli were predicted to have no preferred order of interpretation, such as (8) and (9).

The norming study consisted of two lists each: 24 participants rating the likelihood of the event described in the first sentence as happening before the event that is described in the second sentence, with test items presented in canonical and non-canonical order; and 24 participants rating the likelihood of the event described in the second sentence as happening before the event that is described in the first sentence, again with test items presented in canonical and non-canonical order.

The participants rated the likelihood of the sequences of events using a 7-point scale. Half of the participants rated how likely it is that the event described in the first sentence happens before the event described in the second sentence, by ranking the likelihood on a scale between 1 to 7, 1 being highly likely, and 7 being highly unlikely. The other half of the participants on the other hand rated how likely it is that the event described in the second sentence happens before the event described in the first sentence. The norming procedure was done this way in order to see whether there is symmetry in the preferred order of interpretation: if a stimuli with a high event-order bias has the probable cause appearing in the first sentence, then participants would rate it highly likely that the event described in the first sentence occurs before the event described by the second sentence. Likewise, the other participants would then rate it highly unlikely that the event described in the second sentence occurs before the event described by the first sentence. This mirror-effect is not predicted to occur in stimuli that have a low event-order bias, since those stimuli consist of events that are plausible in either order.

Based on the results of the norming data, a total of 96 stimuli were chosen for the Tagalog experiment. 48 of these were deemed to have a high probability of one described event to occur before the other (having a high event-order bias), while the other 48 were deemed to have no preference for one event occurring before the other (having a low event-order bias). These stimuli were then translated into Tagalog.

\subsection{Tagalog Experiment}

The Tagalog experiment had three experimental factors: perfective or prospective aspect marking on each verb in a pair of sentences; canonical or non-canonical presentational order; and high or low event-order bias. Only the first two factors listed above were crossed within items, resulting in a stimulus set in which half of the items consisted of two-sentence discourse items that had a high event-order bias, and the other half of the items consisted of two-sentence discourse items that had a low event-order bias. 
Aspect marking was crossed with two possible (canonical or non-canonical) presentational orders. In high event-order bias stimuli, the event that is likely to happen first is described in the first sentence for canonically ordered stimuli, but it is described in the second sentence for non-canonically ordered stimuli. In low event-order bias stimuli, variation or presentational order is irrelevant due to the fact that there is no preferred order of the events in the discourse.

In total, each of the 96 stimuli had 8 different versions, depending on the two verbs' aspectual combination and what order the sentences were presented in. The aspectual marking combinations were fully crossed with presentational order. Eight lists of 96 sentence pairs were created, each list with only one version of each 8 stimulus pairs. Each of the 72 native Tagalog participants was assigned to one of the lists and only saw one version of any given stimulus pair.

Each list had between 8 and 10 participants. Participants were instructed to diagram the possible interpretations of the different stimulus pairs by placing the events on a time line. For each stimulus pair, they were given diagrams as in (10).
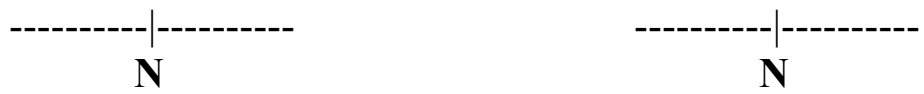

Participants were asked to say in what order they thought the events described in the sentence pair occurred by drawing vertical lines corresponding to the events described by the sentences, either before or after the vertical line for $\mathbf{N}$ (signifying the time of utterance NOW). They were given clear examples of the procedure.

If they interpret the first sentence as happening before the second sentence, then they would put A before B on the timeline. If they interpret the first sentence as happening after the second sentence, they would put B before A on the timeline. Participants were also asked to diagram where the two events are located with respect to the time of utterance. Participants thus had to make three independent judgments: 1) the order of the event described in the first sentence with respect to the time of utterance; 2) the order of the event described in the second sentence with respect to the time of utterance; and 3) the order of the events described in the first and second sentences with respect to each other.

It is worth pointing out that participants had the option to indicate multiple possible interpretations for any given stimulus. Thus, if the stimulus allowed for more than one possible interpretation, they were told to indicate alternative interpretations as well. Participants also had the option to indicate ungrammaticality or infelicity for any given stimulus. If they judged a stimulus as wrong or uninterpretable in any way, there was a way for them to indicate it. All the participants' answers were then summed up, based on the type of stimulus and the participants' judgments.

\section{Results and Discussion}

Even though there is no tense marker in Tagalog, aspect markers receive default temporal interpretation. In other words, sentences that are marked with the 
perfective are more likely to be interpreted as describing events that precede utterance time than sentences that are marked by the prospective. Conversely, sentences that are marked with the prospective are more likely to be interpreted as describing events that follow utterance time (Langacker 1991). This is what is suggested by the results of a binary logistic regression analysis, with aspect of the first sentence (A), aspect of the second sentence (B), presentational order, and event-order bias as predictors. When regressing for the relationship of the event in the first sentence (A) with respect to utterance time (N), the aspect marking of the first sentence emerged as the most important predictor, followed by the aspect of the second sentence, and then presentational order. Event-order bias was found to be non-significant in predicting this relationship, as can be seen in Table 11 . Running a similar test to predict the relationship of the event in the second sentence $(\mathrm{B})$ with respect to utterance time $(\mathrm{N})$ produced similar results, in which the aspect marking of the second sentence was the most important predictor in this relationship. Details can be seen in Table 12.

Relationship of First Sentence to Utterance Time

\begin{tabular}{|lrrrrrr|}
\hline & \multicolumn{1}{c}{$\beta$} & \multicolumn{1}{c}{ S.E. } & \multicolumn{1}{c|}{ Wald's $\chi^{2}$} & df & \multicolumn{1}{c|}{$\mathrm{p}$} & \multicolumn{1}{c|}{$\mathrm{e}^{\beta}$} \\
\hline Aspect of A & -4.490 & 0.084 & 2860.126 & 1 & 0.0001 & 0.011 \\
Aspect of B & -0.761 & 0.077 & 96.704 & 1 & 0.0001 & 0.467 \\
Event-Order Bias & -0.092 & 0.077 & 1.425 & 1 & 0.233 & 0.913 \\
Presentation Order & 0.354 & 0.077 & 21.430 & 1 & 0.0001 & 1.425 \\
Constant & 3.009 & 0.096 & 982.850 & 1 & 0.0001 & 20.274 \\
\hline
\end{tabular}

(12) Relationship of Second Sentence to Utterance Time

\begin{tabular}{|lcrrrrr|}
\hline & $\beta$ & \multicolumn{1}{c}{ S.E. } & Wald's $\chi^{2}$ & df & \multicolumn{1}{c|}{$\mathrm{p}$} & \multicolumn{1}{c|}{$\mathrm{e}^{\beta}$} \\
\hline Aspect of A & -0.921 & 0.075 & 152.070 & 1 & 0.0001 & 0.398 \\
Aspect of B & -4.218 & 0.079 & 2839.351 & 1 & 0.0001 & 0.015 \\
Event-Order Bias & -0.054 & 0.073 & 0.545 & 1 & 0.46 & 0.947 \\
Presentation Order & -0.727 & 0.075 & 94.247 & 1 & 0.0001 & 0.484 \\
Constant & 3.330 & 0.096 & 1193.757 & 1 & 0.0001 & 27.933 \\
\hline
\end{tabular}

The next question to ask is whether world knowledge indeed plays a role in the temporal ordering of sentences in tenseless languages. A binary logistic regression analysis was conducted, with aspect of the first sentence (A), aspect of the second sentence (B), and presentational order as predictors. The data was separated into high and low event-order bias stimuli, and it turned out that the effect of the different predictors varies on whether the stimulus has a high or low event-order bias.

For responses to low event-order bias stimuli, both aspects of A and B equally contribute to predicting the temporal relationship of both events. Presentational order on the other hand is non-significant. This is seen in Table 13. This shows 
that whenever the stimulus pairs have low event-order bias, in other words, if the events that are described by the two sentences are not likely to happen in one particular order, then the two factors that have most influence in predicting the temporal relationship of the two events are the aspect marking of both sentences, as shown by the high Wald statistics for the two aforementioned factors.

\section{Relationship of First Event to Second Event (Low Event-Order Bias)}

\begin{tabular}{|lrrrrrr|}
\hline Low Event-Order Bias & \multicolumn{1}{c}{$\beta$} & \multicolumn{1}{c}{ S.E. } & \multicolumn{1}{c|}{ Wald's $\chi^{2}$} & df & \multicolumn{1}{c|}{$\mathrm{p}$} & $\mathrm{e}^{\beta}$ \\
\hline Aspect of A & -1.942 & 0.082 & 554.399 & 1 & 0.0001 & 0.143 \\
Aspect of B & 1.955 & 0.082 & 563.091 & 1 & 0.0001 & 7.063 \\
Presentation Order & -0.095 & 0.072 & 1.724 & 1 & 0.189 & 0.910 \\
Constant & 0.378 & 0.066 & 32.864 & 1 & 0.0001 & 0.685 \\
\hline
\end{tabular}

\section{Relationship of First Event to Second Event (High Event-Order Bias)}

\begin{tabular}{|lrcrccr|}
\hline High Event-Order Bias & \multicolumn{1}{c}{$\beta$} & S.E. & Wald's $\chi^{2}$ & df & \multicolumn{1}{c|}{$p$} & \multicolumn{1}{c|}{$\mathrm{e}^{\beta}$} \\
\hline Aspect of A & -1.912 & 0.128 & 222.997 & 1 & 0.0001 & 0.148 \\
Aspect of B & 1.851 & 0.126 & 215.072 & 1 & 0.0001 & 6.368 \\
Presentation Order & 4.606 & 0.142 & 1045.670 & 1 & 0.0001 & 100.105 \\
Constant & -2.184 & 0.119 & 337.413 & 1 & 0.0001 & 0.113 \\
\hline
\end{tabular}

On the other hand, for responses to high event-order bias stimuli, presentational order is the most important predictor, followed by the aspect values of both $\mathrm{A}$ and B. In other words, the significance of the various predictors change, depending on whether the stimuli are high or low event-order bias discourses (c.f. Table 14). Thus, event-order bias determines how important each of the other predictors is in establishing the temporal relationship of the two events in the discourse. If a narrative discourse has a low event-order bias, the most important source of temporal interpretation is the aspect marking on the verbs in both sentences. However, if the narrative discourse has a high event-order bias, then aspect marking takes on a smaller role in temporal interpretation. Instead, world knowledge matters more and therefore is the major factor in interpreting the relationship of the two sentences. For high event-order bias stimuli, the Wald statistic for the aspect marking of the two sentences are not as high as the low event-order bias stimuli. Instead, presentational order receives a high Wald statistic, suggesting that speakers tap on to their knowledge of the world and use this knowledge to interpret the discourse's temporal relationship based on that information.

If a stimulus pair has a low event-order bias, then the only cue that influences the temporal relation between events described by the first and second sentence are their aspect markings. However, if a stimulus pair has a high event-order bias, then speakers would compute the probability of the two events happening with respect to each other and interpret events that are probable causes as happening before events that are probable effects, regardless of which event is presented first. 
For stimuli that have a low event-order bias, ordering does not produce a difference in interpretation, due to the fact that neither of the two events has a high probability of occurring before the other event. However, for high event-order bias stimuli, one of the two events has a high probability of occurring before the other, since it may be a probable cause of the other event. If the probable cause is presented as the first sentence, then the stimuli is presented in the canonical order. On the other hand, if the probable cause is presented by the second sentence, then the stimuli is presented in the non-canonical order. Therefore, if the stimuli were presented in the non-canonical order, speakers used their world knowledge to aid in the temporal interpretation and interpreted the second sentence as preceding the first sentence.

\section{Theoretical Implications}

\subsection{Temporal Anaphoricity}

Kamp and Reyle (1993) argue that temporal interpretation involves anaphoricity in tensed languages. This means that the temporal interpretation of events described by sentences is partly influenced by the context in which these sentences occur. They provide as examples the sentences in (15) and (16).

(15) Last week Fred bought his ninth cat. He paid 75 ECU for it.

(16) Bill left the house at a quarter past five. He took a taxi to the station and caught the first train to Bognor.

They argue that the second sentences in these two discourses are interpreted as describing events that occurred in the vicinity of the event described in the first sentence. According to their model, the interpretation of events and states that are described by the subsequent sentences are dependent on the events or states that are described by the previous sentences. A subsequent state overlaps with the time of the previous event or state, while a subsequent event, as seen in examples (15) and (16), is interpreted as occurring in a certain time, which is in turn interpreted as occurring after the time of the preceding events or states. In other words, without the use of explicit temporal adverbials, time always moves forward in this analysis.

However, the analysis of Kamp and Reyle is not directly applicable to Tagalog, since the language has no tense in the first place. In fact, it is not true in the case of Tagalog that the event described by the second sentence refers back to the time of the event described by the first sentence. This is illustrated in the example in (17):

$$
\begin{aligned}
& \text { Na-tumba si Juan. T<in }>\text { ulak ni Bill si Juan. } \\
& \text { PERF-fall SUBJ John }<\text { PERF }>\text { push NSUB Bill SUBJ John } \\
& \text { 'John fell. Bill pushed John.' }
\end{aligned}
$$

In the Tagalog example in (17), it is most likely that the event described in the second sentence is interpreted as happening before the event described in the first sentence. This runs contrary to Kamp and Reyle's analysis. The net effect of 
Kamp and Reyle's view is that the temporal interpretation of discourse is iconic; time always moves forward. Unless there is an explicit signal that the temporal order of the described events is reversed, the second sentence is interpreted as following the first sentence, and the third sentence is interpreted as following the second, and so on. This prediction is not supported by the experimental data from Tagalog, however.

If it were true that temporal interpretation is dependent on anaphoricity, then it should be the case that the event that the second sentence describes is always interpreted as occurring after the event that the first sentence describes. However, we saw that the interpretation of the temporal relationship between A and $\mathrm{B}$ is dependent on other factors aside from presentational order. These other factors include event-order bias, and the aspect marking of the individual sentences themselves. It cannot be argued that anaphoricity effects are still present in an otherwise tenseless language, due to the fact that not all stimuli are interpreted in a canonical order; non-canonical interpretation is possible, which is triggered by the other factors mentioned above.

As explained earlier, speakers use their knowledge of the world to evaluate the relationship of two events in a discourse. For low event-order bias stimulus pairs, the most important factors in temporal interpretation are the aspect marking of both sentences. For high event-order bias stimulus pairs on the other hand, the speakers' knowledge of the world is the most important factor in temporal interpretation, since depending on which order a given stimulus is presented in, the speakers will evaluate differently how the two events are related, i.e., probable causes will be interpreted as happening before probable effects. Thus, world knowledge plays a factor in temporal interpretation as well, overriding the information given by aspect marking.

Therefore, it is not true that anaphoricity always plays a role in the temporal interpretation of ordered sentences. If anaphoricity was the main component in temporal interpretation, then there would be no cases of non-canonical interpretation of discourse. Regardless as to whether the stimuli has a high or low eventorder bias, the second sentence would always be interpreted as occurring after the first sentence, since the interpretation of the second sentence is dependent on the first. However that is not always the case. Instead, what we can see here is that world knowledge plays the most important role in the temporal ordering of sentences for tenseless languages, a fact that contradicts the anaphoricity hypothesis. If world knowledge does not provide cues, as in the case of low event-order bias pairs, then it is aspect markers that are present in the sentences and their default tense interpretation that are the source of information for temporal interpretation. And if the aspect markers are the same in low event-order bias pairs, then the interpretation would be based solely on presentational order; i.e., the event described by the first sentence is interpreted as occurring before the event described by the second sentence. 
Jeruen E. Dery

\subsection{The Role of World Knowledge}

It has been claimed early on by Dowty (1986) that temporal interpretation depends on three different factors: 1) a semantic analysis of Aktionsarten using interval semantics, as expounded in Dowty (1979); 2) a single principle for the interpretation of successive sentences in a discourse, which does not make reference to Aktionsarten; and 3) Gricean conversational implicatures and "common sense" reasoning based on world knowledge. He then presents an interpretation principle for temporal discourse, which takes into account the type of Aktionsart the predicate has, and the type of (grammatical) aspect that they are marked for. Using these pieces of information, he posits the Temporal Discourse Interpretation Principle (Dowty 1986).

According to the interpretation principle, it is only possible to have reverse temporal interpretation when definite time adverbials are used that would explicitly signal reversal of temporal interpretation. Dowty admits that the principle has to be modified to explain elaborating narratives and he also mentions that world knowledge is essential, but he does not describe how it affects temporal interpretation.

More recently, numerous scholars have suggested that world knowledge, in the form of coherence relations, play a role in ordering events in discourse. A model of temporal relations as a by-product of reasoning about coherence relations has been put forward in Lascarides and Asher (1993) and Asher and Lascarides (2003). Kehler (2002) on the other hand puts forth an account of temporal ordering that combines tense information and coherence relations. An important question to ask then is to what extent coherence relations influence temporal ordering.

In the experiment that I conducted, the stimuli were designed so that half of them would be high event-order bias stimuli, and the other half low event-order bias stimuli. If coherence relations play a part in temporal interpretation, there should be an observable difference in how described events are ordered between these two groups.

It was seen that participants were sensitive to world knowledge of cause and effect for high event-order bias stimuli. Aspect marking is still significant, but if one looks at the Wald statistic for aspect marking on high event-order bias pairs in Tables 13 and 14, it is clear that it is not as significant as aspect marking for low event-order bias pairs. If sensitivity to the knowledge of probable causes and probable effects matters highly for this type of stimuli, it suggests that speakers use their world knowledge information to determine what type of coherence relation can be established, and then the temporal relation between events. Crucially, this analysis of temporal ordering does not depend on temporal anaphora, since reversal of reference time ordering is possible. Theories of temporal anaphora seem irrelevant to handle ordering of events in Tagalog.

\section{Conclusion}

This paper has shown that in a tenseless language such as Tagalog, different factors influence the temporal interpretation of discourse. A three-factor experi- 


\section{Temporal Interpretation and Tenselessness}

ment was conducted to test and see which factors make a larger contribution to the task of temporally ordering events described by pairs of sentences. The results of the experiment show that there are more factors that are involved in interpreting the temporal relationships that exist between events. Aside from aspectual information that is explicitly marked in the predicates, world knowledge influences temporal interpretation as well.

It was shown how world knowledge and coherence relations are only part of what determines temporal interpretation. The data support the claim that coherence relations play a role in temporal interpretation. However, it was also pointed out that temporal constraints put forth by coherence relations can be overridden, as when the information coming from aspect markers contradicts the possible coherence relation that can be established between the two events, and when some participants ignored the constraints that coherence relations provided. Coherence relations most frequently dictate what the available temporal interpretation is, but not always.

In conclusion, the results of the Tagalog experiment suggest that there is a hierarchy of importance of the different factors that influence temporal interpretation. Lack of tense in Tagalog paved way for other factors to play a role in temporal interpretation. Coherence relations constrain the possible temporal interpretations of a given discourse, depending on their aspect marking. Finally, if coherence relations do not make a prediction, one can assign default tense interpretations to aspect marking, which can be a source of the temporal interpretation as well, as long as the coherence relation does not contradict the default interpretation.

We cannot generalize the findings of this study to other tenseless languages. Further experimental research must be done in other tenseless languages to see whether the same conclusions attained by examining the Tagalog data hold, since the pattern exhibited here may not be true to other tenseless languages. In addition, an experimental comparison between tensed and tenseless languages must also be done.

\section{References}

Asher, Nicholas, and Alex Lascarides. 2003. Logics of conversation. Cambridge: Cambridge University Press.

Comrie, Bernard. 1976. Aspect: An introduction to the study of verbal aspect and related problems. London: Cambridge University Press.

Dowty, David. 1979. Word meaning and Montague Grammar. Dordrecht: Reidel.

Dowty, David. 1986. The effects of aspectual class on the temporal structure of discourse: Semantics or pragmatics? Linguistics and Philosophy 9(1):37-61.

Kamp, Hans, and Uwe Reyle. 1993. From discourse to logic. Dordrecht: Kluwer.

Kehler, Andrew. 2002. Coherence, reference, and the theory of grammar. Stanford, CA: CSLI Publications. 
Jeruen E. Dery

Langacker, Ronald. 1991. Foundations of Cognitive Grammar (Vol. 2). Stanford, CA: Stanford University Press.

Lascarides, Alex, and Nicholas Asher. 1993. Temporal interpretation, discourse relations, and common sense entailment. Linguistics and Philosophy 16(5):437-493.

Partee, Barbara. 1973. Some structural analogies between tenses and pronouns in English. Journal of Philosophy 70(18):601-609.

Partee, Barbara. 1984. Nominal and temporal anaphora. Linguistics and Philosophy 7(3):243-286.

Jeruen E. Dery

Dept. of Linguistics

609 Baldy Hall

University at Buffalo - SUNY

Buffalo, NY 14260

jedery@buffalo.edu 